\title{
The Minimal System of Defining Relations of the Free Modular Lattice of Rank 3 and Lattices Close to Modular One
}

\author{
Alexander G. Gein, Mikhail P. Shushpanov* \\ Ural Federal University, Russia \\ *Corresponding Author: mikhail.shushpanov@gmail.com
}

Copyright $\odot 2014$ Horizon Research Publishing All rights reserved.

\begin{abstract}
We construct the system of 11 defining relations for the 3-generated free modular lattice. This system is proved to be minimal. Systems of defining relations for lattices close to modular one are studied.
\end{abstract}

Keywords Free modular lattices, Defining relations

\section{Introduction and Statement of Results}

Recall that the rank of a free algebra of a variety is the number of its free generators. Let $A$ denote the free lattice of rank 3 in the variety of the modular lattices and let $F$ be the free lattice of rank 3 in the variety of all lattices. Let $f, g, h$ be free generators of $F$, and let $\phi$ be a homomorphism from $F$ onto $A$. Then the elements $a=\phi(f), b=\phi(g)$, and $c=\phi(h)$ are free generators of the lattice $A$. Defining relations of the lattice $A$ in the variety of all lattices are considered in [1] and [2]. Namely, one of the results of [1] can be interpreted as the statement that the lattice $A$ can be defined by 21 relations. In [2] it was noted that the set of these relations is not a minimal set, namely, 15 from these 21 relations were selected and the 15 they were proved to form a minimal set of the defining relations of $A$. Note that in [1] it was presented the set of 7 defining relations of the free distributive lattice of rank 3 . In [3] this set is proved to be minimal.

The main result of the present paper is that we have found a set of 11 defining relations for the lattice $A$. Note that this set is not a subset of the defining relations from [1].

We list these relations:

$$
\begin{aligned}
& (a \vee b) \wedge(a \vee c) \wedge(b \vee c)= \\
& =((a \wedge(b \vee c)) \vee(c \wedge(a \vee b))) \wedge \\
& \wedge((b \wedge(a \vee c)) \vee(c \wedge(b \vee a))) \wedge \\
& \wedge((a \wedge(c \vee b)) \vee(b \wedge(a \vee c)))
\end{aligned}
$$

$$
\begin{gathered}
(a \wedge b) \vee(a \wedge c) \vee(b \wedge c)= \\
=((a \vee(b \wedge c)) \wedge(c \vee(a \wedge b))) \vee \\
\vee((b \vee(a \wedge c)) \wedge(c \vee(b \wedge a))) \vee \\
\vee((a \vee(c \wedge b)) \wedge(b \vee(a \wedge c))) \\
(a \vee(b \wedge c)) \wedge(b \vee c)=(a \wedge(b \vee c)) \vee(b \wedge c) \\
(b \vee(c \wedge a)) \wedge(c \vee a)=(b \wedge(c \vee a)) \vee(c \wedge a) \\
(c \vee(a \wedge b)) \wedge(a \vee b)=(c \wedge(a \vee b)) \vee(a \wedge b) \\
(a \vee b) \wedge(a \vee c)=a \vee((a \vee b) \wedge(a \vee c) \wedge(b \vee c)) \\
(b \vee a) \wedge(b \vee c)=b \vee((a \vee b) \wedge(a \vee c) \wedge(b \vee c)) \\
(c \vee a) \wedge(c \vee b)=c \vee((a \vee b) \wedge(a \vee c) \wedge(b \vee c)) \\
(a \wedge b) \vee(a \wedge c)=a \wedge((a \wedge b) \vee(a \wedge c) \vee(b \wedge c)) \\
(b \wedge a) \vee(b \wedge c)=b \wedge((a \wedge b) \vee(a \wedge c) \vee(b \wedge c)) \\
(c \wedge a) \vee(c \wedge b)=c \wedge((a \wedge b) \vee(a \wedge c) \vee(b \wedge c))
\end{gathered}
$$

Denote the set of these relations by $\rho$.

Theorem 1. The lattice generated by the elements $a, b, c$ subject to the defining relations $\rho$ is isomorphic to the free modular lattice of rank 3. If a set $\sigma$ of relations is strictly contained in $\rho$, then the lattice generated by $a, b, c$ subject to the defining relations $\sigma$ is not modular.

In light of this theorem there is natural interest in lattices generated by $a, b, c$ subject to one or another subset $\sigma$ of the defining relations from the set $\rho$. In particular, how much they differ from the free modular lattice of rank 3. In the following theorem the answer is given for $\sigma$ consisting of relations (1) and (2).

Theorem 2. The lattice generated by the elements $a, b, c$ subject to the defining relations (1) and (2) is isomorphic to the lattice represented on Figure 1.

In reality, Theorem 2 makes it easy to get a description of the lattice generated by the elements $a, b$ and $c$ subject to a set $\sigma$ of relations when $\{(1),(2)\} \subset \sigma \subset \rho$.

Theorem 1 was announced in [4] without proof. 


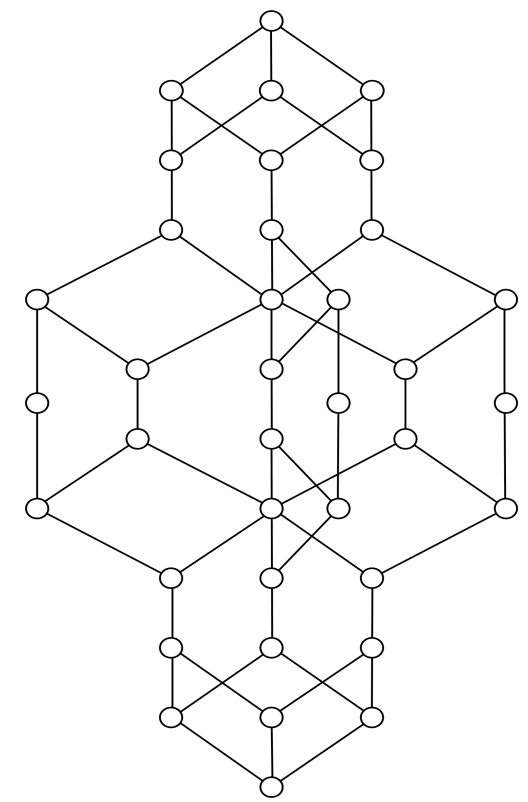

Figure 1. The lattice which is close to modular one

\section{Proofs}

Let $a, b, c$ be any elements of a lattice. Further we use the following notation:

$$
\begin{aligned}
& a_{1}=(a \vee(b \wedge c)) \wedge(b \vee c) ; \\
& a_{2}=(a \wedge(b \vee c)) \vee(b \wedge c) ; \\
& b_{1}=(b \vee(c \wedge a)) \wedge(c \vee a) ; \\
& b_{2}=(b \wedge(c \vee a)) \vee(c \wedge a) ; \\
& c_{1}=(c \vee(a \wedge b)) \wedge(a \vee b) ; \\
& c_{2}=(c \wedge(a \vee b)) \vee(a \wedge b) ; \\
& t=(a \vee b) \wedge(a \vee c) \wedge(b \vee c) ; \\
& s=(a \wedge b) \vee(a \wedge c) \vee(b \wedge c) .
\end{aligned}
$$

Lemma 1. The following inequalities hold in any lattice:

$$
\begin{aligned}
& s \leq a_{2} \leq a_{1} \leq t \\
& s \leq b_{2} \leq b_{1} \leq t \\
& s \leq c_{2} \leq c_{1} \leq t
\end{aligned}
$$

Proof. The distributive inequality [5, Lemma I.4.9] shows that

$$
(a \vee(b \wedge c)) \wedge(b \vee c)) \leq(a \vee b) \wedge(a \vee c) \wedge(b \vee c)
$$

i.e. $a_{1} \leq t$. Passing on to the dual lattice we get $s \leq a_{2}$. At the same time, since $b \wedge c \leq b \vee c$ the same inequality shows that

$$
(a \wedge(b \vee c)) \vee(b \wedge c) \leq(a \vee(b \wedge c)) \wedge(b \vee c),
$$

i.e. $a_{2} \leq a_{1}$. Consequently, the inequalities $s \leq a_{2} \leq$ $a_{1} \leq t$ hold in any lattice.

Other inequalities can be derived by cyclic substitutions of elements $a, b$ and $c$.

Lemma 2. Let $L$ be a lattice generated by $a, b, c$. If relation (2) holds for elements $a, b, c$ then

$$
\begin{aligned}
& (a \vee(b \wedge c)) \wedge(c \vee(a \wedge b)=s \\
& (a \vee(b \wedge c)) \wedge(b \vee(a \wedge c)=s \\
& (b \vee(a \wedge c)) \wedge(c \vee(a \wedge b)=s .
\end{aligned}
$$

Proof. It is clear that $a \vee(b \wedge c) \geq s$ and $c \vee(a \wedge b) \geq s$, therefore $(a \vee(b \wedge c)) \wedge(c \vee(a \wedge b) \geq s$. But from (2) it follows that $(a \vee(b \wedge c)) \wedge(c \vee(a \wedge b)) \leq s$, hence the first equality holds. Other equalities are derived by cyclic substitutions of elements $a, b$ and $c$.

From (1) the statement dual of Lemma 2 is proved similarly.

Let $L$ denote the lattice generated by $a, b, c$. And the defining relations (1) and (2) holds for elements $a, b, c$ in $L$. Let us consider the following subsets of the lattice $L:$

$M_{1}=\{a \vee b \vee c, a \vee c, a \vee b, b \vee c,(a \vee b) \wedge(a \vee c)$, $(a \vee c) \wedge(b \vee c),(a \vee b) \wedge(b \vee c), a \vee t, c \vee t, b \vee t, t\}$ $M_{2}=\{a \wedge b \wedge c, a \wedge c, a \wedge b, b \wedge c,(a \wedge b) \vee(a \wedge c)$, $(a \wedge c) \vee(b \wedge c),(a \wedge b) \vee(b \wedge c), a \wedge s, b \wedge s, c \wedge s, s\} ;$

$M_{a}=\left\{a \vee(b \wedge c), a, a_{1}, a_{2}, a \wedge(b \vee c)\right\} ;$

$M_{b}=\left\{b \vee(a \wedge c), b, b_{1}, b_{2}, b \wedge(a \vee c)\right\}$; $M_{c}=\left\{c \vee(b \wedge a), c, c_{1}, c_{2}, c \wedge(b \vee a)\right\}$.

Lemma 3. The set $M_{1} \cup M_{2} \cup M_{a} \cup M_{b}$ is a sublattice of the lattice $L$.

Proof. Since the set $M_{1} \cup M_{2} \cup M_{a} \cup M_{b}$ is self-dual and relations (1) and (2) are dual to one another it is sufficient to check on the closure of this set under the operation $\wedge$. Also it is clear that we have to consider those pairs of elements that are not known to be comparable. The check will be performed in several steps.

The set $M_{1} \cup M_{2} \cup M_{a} \cup M_{b}$ is shown on the Figure 2 . Here we don't denote the elements $(b \vee c) \wedge(a \vee c)$ and $(b \wedge c) \vee(a \wedge c)$ to do not overload the figure. If $x \geq y$ for some $x$ and $y$ from the lattice $L$ they are joined by a line and the element $x$ is above than $y$. The inequalities between $a_{1}$ and $a_{2}, b_{1}$ and $b_{2}, c_{1}$ and $c_{2}, t$ and $s$ have been proved in Lemma 1 . Other inequalities are well known or checked easy. But we do not guarantee as yet that all elements shown in this diagram are different.

Let us carry out the necessary calculations.

1) $(a \vee c) \wedge((a \vee b) \wedge(b \vee c))=t$. It means that $x \wedge y=t$ for any element $x$ from the interval $[t, a \vee c]$ and for any element $y$ from the interval $[t,(a \vee b) \wedge(b \vee c)]$.

2) $(a \vee(b \wedge c)) \wedge(b \vee c)=a_{1}$. Then $(a \vee(b \wedge c)) \wedge x=a_{1}$ for any element $x$ from the interval $\left[a_{1}, b \vee c\right]$.

3) $a \wedge x=a \wedge(b \vee c)$ for any element $x$ from the interval $[a \wedge(b \vee c), b \vee c]$

4) $(a \vee(b \wedge c)) \wedge(b \vee(a \wedge c))=s$ by Lemma 2. It means that the set $\left\{a \vee(b \wedge c), a_{1}, a_{2}, s, b_{2}, b_{1}, b \vee(a \wedge c)\right\}$ is closed under the operation $\wedge$.

5) The equality $(a \vee(b \wedge c)) \wedge(b \vee(a \wedge c))=s$ implies $(a \vee(b \wedge c)) \wedge b=s \wedge b$. Then the set $\left\{a \vee(b \wedge c), a_{1}, a_{2}, s\right.$, $b \vee s, b \wedge(a \vee c), b\}$ is closed under the operation $\wedge$.

6) $x \wedge y=a \wedge b$ for any element $x$ from the interval $[a \wedge b, a]$ and for any element $y$ from the interval $[a \wedge b, b]$.

7) $a \wedge(c \wedge s)=a \wedge c$. It means the closure of the set $\{a, a \wedge(b \wedge c), a \wedge s,(a \wedge b) \vee(a \wedge c), a \wedge c,(a \wedge c) \vee(b \wedge c), c \wedge s\}$ under the operation $\wedge$.

8) $(b \wedge c) \wedge x=a \wedge b \wedge c$ for any element $x$ from the interval $[a \wedge b \wedge c, a]$.

The roles of $a$ and $b$ in $M_{1} \cup M_{2} \cup M_{a} \cup M_{b}$ are interchangeable, and so are the roles of $a, b$ and $c$ in $M_{1} \cup M_{2}$. Hence the verifications made above suffice to claim the closeness of the set $M_{1} \cup M_{2} \cup M_{a} \cup M_{b}$ under the operation $\wedge$.

In the proof of Lemma 4 we use the following generalalgebraic observation. Let $U$ be an universal algebra 
with binary operation $*$ and let $\left\{K_{i} \mid i \in I\right\}$ be a family of subsets of the algebra $U$. Suppose that for any pair of subsets $K_{i}$ and $K_{j}$ (which are not necessarily different) from this family, their union $K_{i} \cup K_{j}$ is closed under this operation. Then $\cup\{K i \mid i \in I\}$ is also closed under the operation $*$ too. Indeed, if $x, y \in \cup\left\{K_{i} \mid i \in I\right\}$ then there exist $i$ and $j$ that $x \in K_{i}$ and $y \in K_{j}$. Hence $x * y \in K_{i} \cup K_{j} \subseteq \cup\left\{K_{i} \mid i \in I\right\}$.

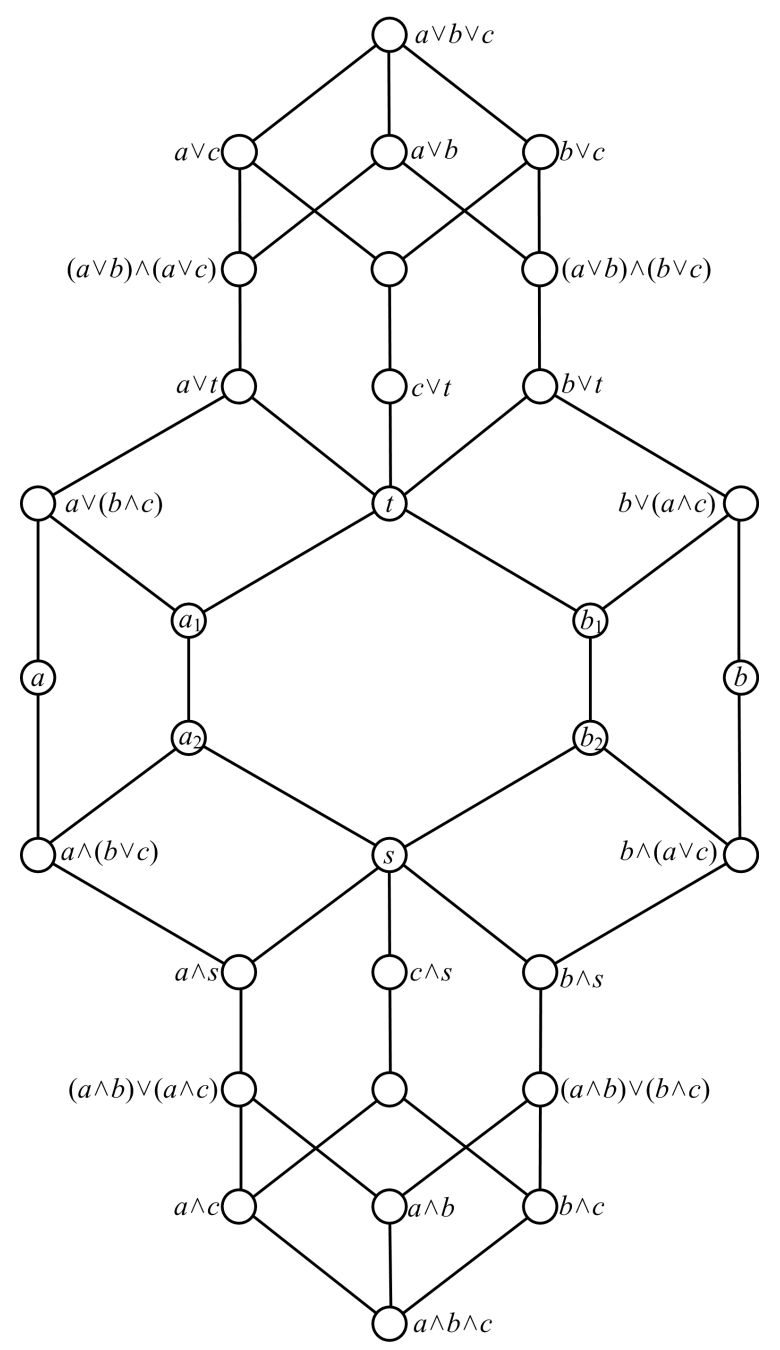

Figure 2. The set $M_{1} \cup M_{2} \cup M_{a} \cup M_{b}$

Lemma 4. The set $M_{1} \cup M_{2} \cup M_{a} \cup M_{b} \cup M_{c}$ is a sublattice of the lattice $L$.

Proof. Let $P=M_{1} \cup M_{2} \cup M_{a}, Q=M_{1} \cup M_{2} \cup M_{b}$ and $R=M_{1} \cup M_{2} \cup M_{c}$. By Lemma 3 the sets $P \cup Q$, $P \cup R, Q \cup R$ are closed under $\wedge$ and $\vee$. Consequently the set $P \cup Q \cup R$ is closed under these operations too, i.e. this set is a sublattice of the lattice $L$.

Below we will use the following remark well known from general algebra. Let $L_{1}$ and $L_{2}$ be lattices with the same set of generating elements and let $\tau_{1}$ and $\tau_{2}$ be sets of defining relations, respectively, for these lattices. In this case if $\tau_{1} \subseteq \tau_{2}$ then the identity mapping of generating elements of lattice $L_{1}$ on generating elements of lattice $L_{2}$ can be continued to a surjective homomorphism of $L_{1}$ on $L_{2}$.

Before proving Theorem 2 let us consider the lattice which is mentioned in this theorem. For convenience we denote it by $L_{1}$. It has 37 elements. Also it is obvious that this lattice is generated by three elements. On Figure 3 we have denoted them as $a, b$ and $c$. Other elements of the lattice have been evaluated by this generators and they have been marked by corresponding formulas apart from elements $c \vee(a \wedge b), c \wedge(a \vee b)$, $(a \vee c) \wedge(b \vee c)$ and $(a \wedge c) \vee(b \wedge c)$. As above $a_{1}, a_{2}$, $b_{1}, b_{2}, c_{1}, c_{2}, t$ and $s$ denote the elements with respect to formulas $(12)-(19)$.

Of course, for some elements of $L_{1}$ we can write also other variants of their representations by the generating elements $a, b$ and $c$. For example, $t=a_{2} \vee b_{2}=a_{2} \vee c_{2}=$ $b_{2} \vee c_{2}$. Each of these equations is a certain relation between the generating elements $a, b, c$ of the lattice $L_{1}$.

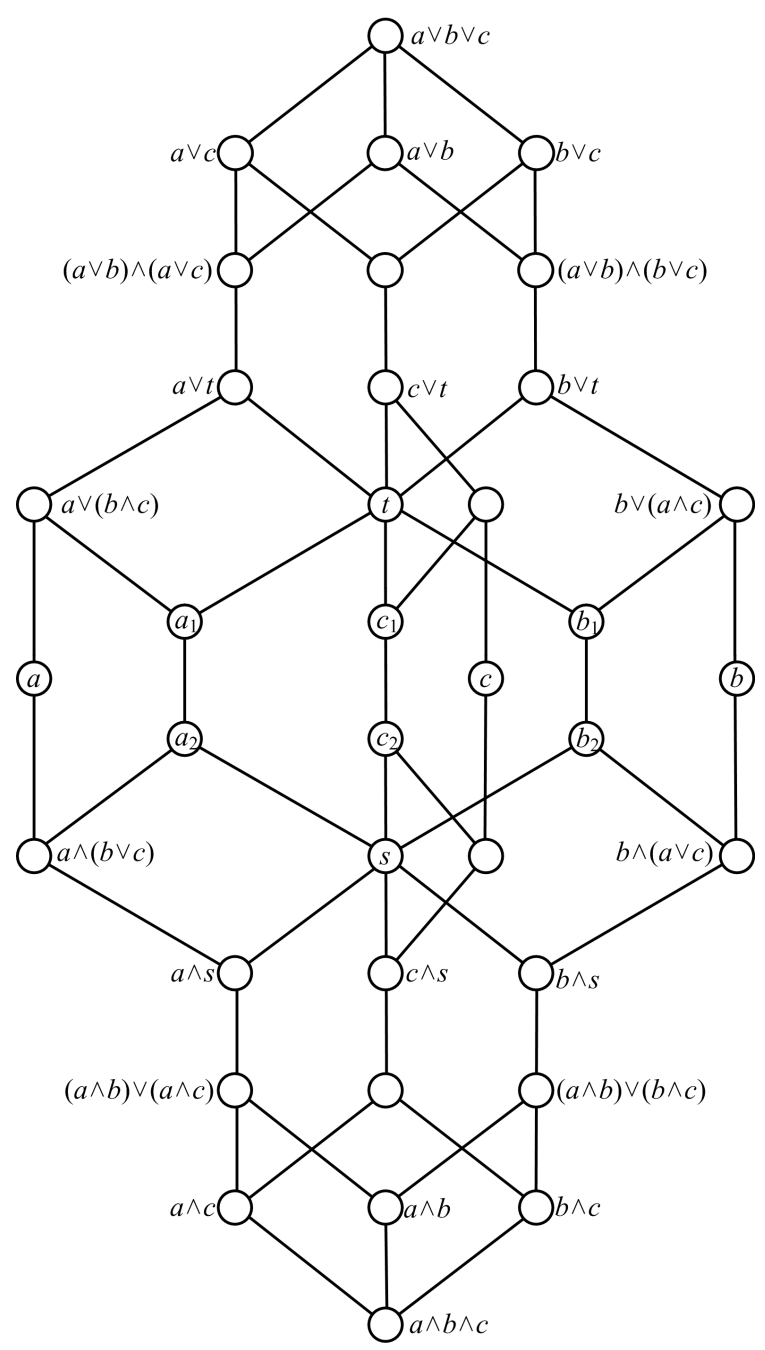

Figure 3. The set $M_{1} \cup M_{2} \cup M_{a} \cup M_{b} \cup M_{c}$

Let $\tau$ denote the set of all relations of the generated elements $a, \mathrm{~b}$ and c of the lattice $L_{1}$.

Proof of Theorem 2. Let $L_{2}$ be a lattice generated by elements $\mathrm{a}, \mathrm{b}$ and $\mathrm{c}$ with the defining relations (1) and (2). The lattice $L_{1}$ is the homomorphic image of the lattice $L_{2}$ since relations (1) and (2) are contained in $\tau$. Consequently there are at least 37 different elements in the lattice $L_{2}$.

In the same time by Lemma 4 the set

$$
M_{1} \cup M_{2} \cup M_{a} \cup M_{b} \cup M_{c}
$$

is a sublattice of the lattice $L_{2}$. This sublattice contains the elements $a, b$ and $c$, hence $L_{2}$ contains at most 37 elements. It means that the lattices $L_{1}$ and $L_{2}$ are isomorphic. 
Proof of Theorem 1. Let $L_{3}$ be the lattice generated by elements $a, b$ and $c$ with the defining relations $\rho$. Let $\phi$ be the identity mapping from the generating elements $a, b, c$ of $L_{2}$ onto the generating elements $a, b, c$ of $L_{3}$. Since the relations (1) and (2) are contained in $\rho$, the mapping $\phi$ can be continued to a surjective homomorphism from $L_{2}$ onto $L_{3}$. This homomorphism identifies elements at least in 9 pairs: $a_{1}$ and $a_{2}, b_{1}$ and $b_{2}, c_{1}$ and $c_{2}$ because $(3)-(5)$ are true, $a \vee t$ and $(a \vee b) \wedge(a \vee c)$, $b \vee t$ and $(b \vee a) \wedge(b \vee c), c \vee t$ and $(c \vee a) \wedge(c \vee b)$ because (6) - (8) are true, and also three pairs of dual elements because $(9)-(11)$ are true. This means that the number of elements of $L_{3}$ is at most 28 .

Also all relations from $\rho$ are true for any modular lattice (e.g. [5]). Therefore there exists surjective homomorphism $\varphi$ from lattice $L_{3}$ onto lattice $A$ that contains exactly 28 elements. Consequently $\varphi$ is an isomorphism.

For the proof of the second statement, for any set $\sigma$ of defining relations obtained from $\rho$ by removing one of its elements we construct a non-modular lattice satisfying the relations in $\sigma$. For example, the lattice in Figure 4 satisfies the relations (2) - (11) but it does not satisfy the relation (1).

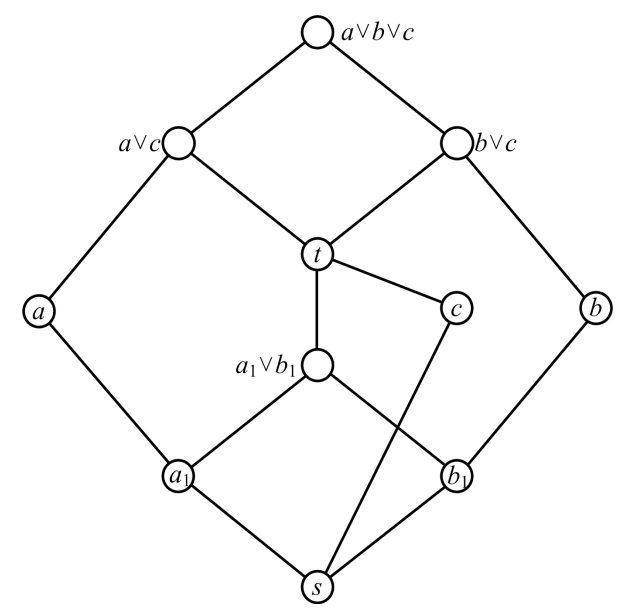

Figure 4. The lattice which does not satisfy the relation (1)

The lattice in Figure 5 is not modular and satisfies the relations (1), (2), and (4) - (11) but it does not satisfy the relation $(3)$.

The lattice in Figure 6 satisfies the relations (1) (7) and (9) - (11) but it does not satisfy the relation (8). The element $(b \vee c) \wedge(a \vee c)$ is not denoted to do not overload the figure.

All other examples can be obtained from the ones presented in Figures $4-6$ by a suitable renaming of elements and passing, if necessary, to dual lattices. To show independence of the relation (3) from the other 10 relations it is possible to give an example of lattice with a smaller number of elements (for example, such as in [4]). But the lattice of Figure 5 is the lattice generated by the elements a, b, c subject to the defining relations (1), (2), (4) - (11). That is why it is interesting. The proof is similar to the proof of Theorem 1 using the result of Theorem 2. In this regard Figure 6 gives the lattice generated by the elements a, b, c subject to the defining relations $(1)-(7),(9)-(11)$.

Using Theorem 2 it is easy, in the same way, to obtain the lattice generated by the elements $a, b, c$ subject to

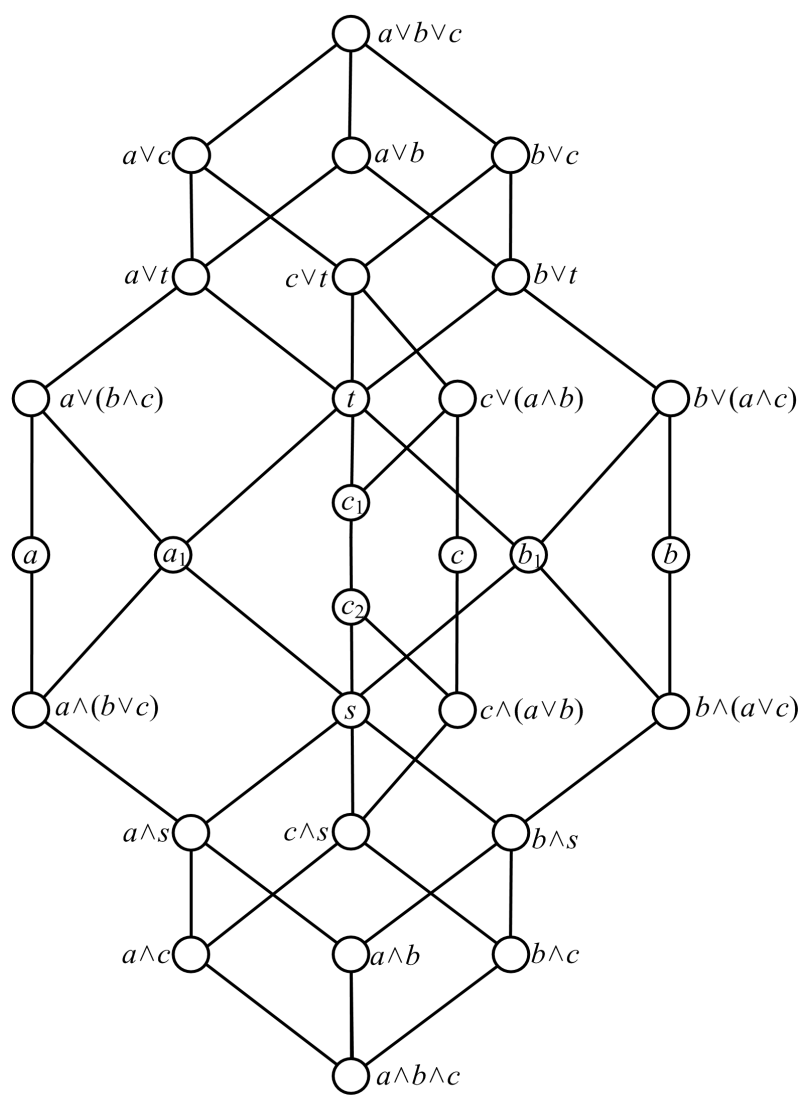

Figure 5. The lattice which does not satisfy the relation (3)

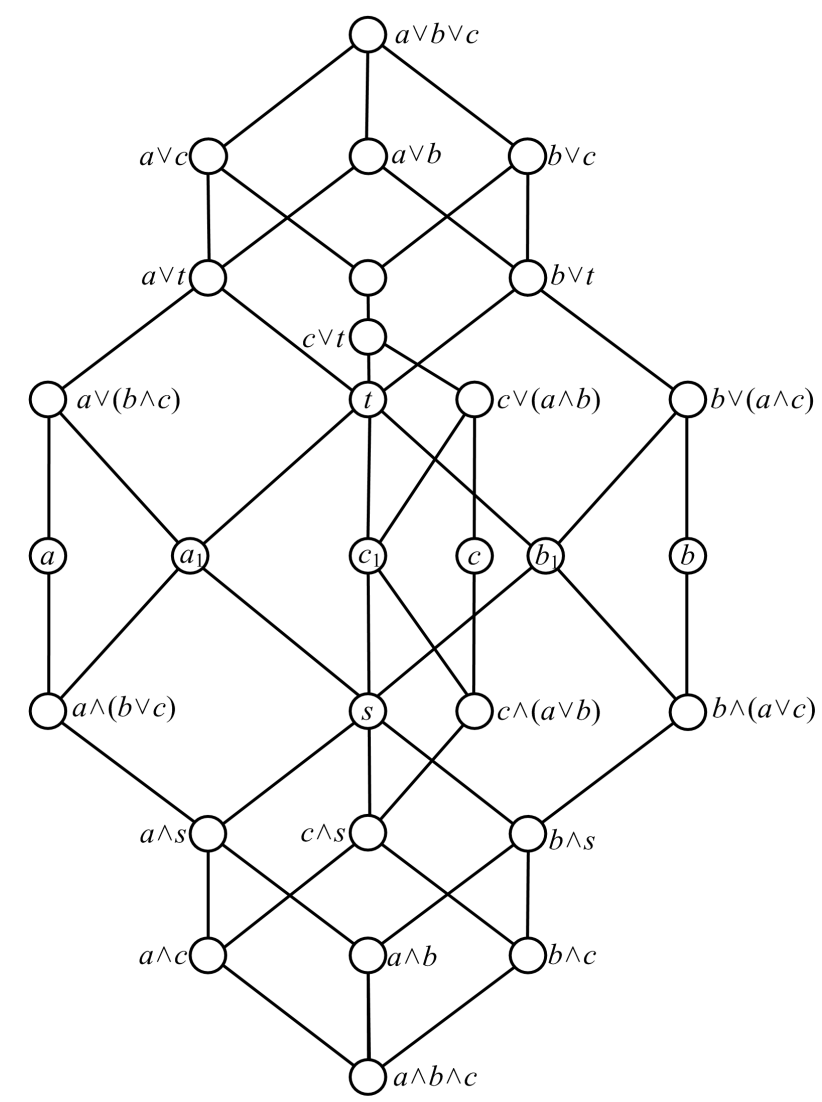

Figure 6. The lattice which does not satisfy the relation (8) 
the set of defining relations $\sigma$ where $\{(1),(2)\} \subset \sigma \subset \rho$.

\section{Discussion}

We note that in connection with this paper it would be interesting to find out whether there exists a set of defining relations for the free modular lattices of rank 3 in which the number of relations will be less than 11 .

Although the defining relations (1) and (2) gives the nonmodular lattice it is finite. Whether the lattice generated by the elements $a, b, c$ subject to the defining relations (2) - (11) finite? It is natural to expect a negative response but it is unknown to us.

Lemma 2 shows that the relation (2) considered as the identity is equivalent to the identity $(x \wedge y) \vee(x \wedge z) \vee$ $(y \wedge z)=(y \vee(x \wedge z)) \wedge(z \vee(x \wedge y))$ In turn this identuty is equivalent to the modularity of a latiice. Indeed if $x \leq y$ then $(y \vee(x \wedge z)) \wedge(z \vee(x \wedge y))=y \wedge(z \vee x)$ and $(x \wedge y) \vee(x \wedge z) \vee(y \wedge z)=x \vee(y \wedge z)$. It means that the modularity follows from the identity. Conversely if a lattice is modular then the equalities $(x \wedge y) \vee(x \wedge z) \vee(y \wedge$ $z)=(y \wedge(z \vee(x \wedge y)) \vee(x \wedge z)=(y \vee(x \wedge z)) \wedge(z \vee(x \wedge y))$ follow from inequalities $y \wedge y \leq x$ and $y \wedge z \leq z \leq$ $z \vee(x \wedge y)$. In the same time the variety $\mathcal{V}$ generated by the free modular lattice of rank 3 is a subvariety of all modular lattices. This variety is clear to be contained in the variety $\mathcal{W}$ generated by the lattice on Figure 1. Since the lattice on Figure 1 is close to the free modular lattice of rank 3 we can expect that in the lattice of varieties the interval $[\mathcal{V}, \mathcal{W}]$ is not very long.

\section{Acknowledgements}

We would like to express our thanks to Lev Shevrin for the discussions that contributed to significant improvements of the text.

\section{REFERENCES}

[1] O. Ore. Remarks on structures and group relations, Vierteljahrsschrift der Naturforschenden Gesellsachaft in Zurich 85 (32), 1 - 4 (1940).

[2] Z. Ladzianska. Modular substructures of an structure, Mat. časop. 24 (1), 81 - 83 (1974).

[3] M. Kolibiar. Distributive sublattices of a lattice, Proc. Amer. Math. Soc. 34 (2), 359 - 364 (1972).

[4] A.G. Gein, M.P. Shushpanov. Defining relations of a free modular lattice of rank 3 - Russian Mathematics, N 10 (2013), pp. 59 - 61.

[5] G. Grätzer. General Lattice Theory. Akademie-Verlag, Berlin, 1978. 